\title{
Implementing a New Dose-response Model for Estimating Infection Probability of Campylobacter Jejuni based on the Key Events Dose-response Framework
}

\author{
Hiroki Abe \\ Hokkaido University \\ Kohei Takeoka \\ Hokkaido University \\ Yuto Fuchisawa \\ Hokkaido University \\ Kento Koyama \\ Hokkaido University \\ Shigenobu Koseki ( $\square$ koseki@bpe.agr.hokudai.ac.jp ) \\ Hokkaido University
}

\section{Research Article}

Keywords: ingested pathogenic bacteria, foodborne pathogens, mechanistic , uman gastrointestinal environment

Posted Date: January 25th, 2021

DOl: https://doi.org/10.21203/rs.3.rs-148212/v1

License: (c) (1) This work is licensed under a Creative Commons Attribution 4.0 International License.

Read Full License

Version of Record: A version of this preprint was published at Applied and Environmental Microbiology on September 28th, 2021. See the published version at https://doi.org/10.1128/AEM.01299-21. 
1 Implementing a new dose-response model for estimating infection probability of

2 Campylobacter jejuni based on the key events dose-response framework

3

4 Hiroki Abe, Kohei Takeoka, Yuto Fuchisawa, Kento Koyama, Shigenobu Koseki*

5

6 Graduate School of Agricultural Science, Hokkaido University, Kita-9, Nishi-9, Kita-ku,

7 Sapporo 060-8589, Japan

8

$9 \quad$ *Corresponding author: Phone/fax: +81 117062552

10 E-mail address: koseki@bpe.agr.hokudai.ac.jp (S. Koseki) 


\section{Abstract}

12 Understanding the dose-response relationship between ingested pathogenic bacteria and

13 infection probability is a key factor for appropriate risk assessment of foodborne pathogens.

14 The objectives of this study were to develop and validate a novel mechanistic dose-

15 response model for Campylobacter jejuni and simulate the underlying mechanism of

16 foodborne illness during digestion. Bacterial behavior in the human gastrointestinal

17 environment, including gastric reductions, transition to intestines, and invasion to intestinal

18 tissues, was described using a Bayesian statistical model based on the reported

19 experimental results of each process while considering physical food types (liquid or solid)

20 and host age (young or elderly). Combining the models in each process, the relationship

21 between pathogen intake and the cell invasion probability of $C$. jejuni was estimated and

22 compared with reported epidemiological dose-response relationships. Taking food types

23 into account, estimations of the cell invasion probability of $C$. jejuni successfully described

24 the reported dose-response relationships from substantial accidents. The developed

25 calculation framework is thus potentially applicable to other pathogens to quantify the

26 dose-response relationship from experimental data obtained from digestion. 
Introduction

29 Dose-response models play an important role in quantitative microbial risk assessment

30 (QMRA) for food. While the exposure assessment of the QMRA helps predict the bacterial

31 response during processing and distribution of foods, dose-response models play a key role

32 in risk characterization, which estimates the probability of illness or infection from

33 pathogen intake counts derived from the exposure assessment. Three main approaches for

34 developing dose-response relationships of foodborne pathogens are available: testing with

35 human volunteers, animal tests, and epidemiological estimation from outbreak data.

36 Although each approach has strengths and limitations, all three approaches have substantial

37 uncertainty owing to the inherent variability in the pathogen, host, and food vehicle. In

38 addition, it is generally difficult to collect data at a low pathogen concentration to which a

39 person is exposed, and it is difficult to collect relevant data during the response ${ }^{1}$.

40 An alternative approach has been suggested for establishing dose-response models; the

41 Key Events Dose-Response Framework (KEDRF) ${ }^{2}$. KEDRF is an approach based on

42 important infection mechanisms causing foodborne illness, called key events, and available

43 data from digestive systems to gain insight into dose-response relationships. As the method

44 for estimating the dose-response relationship is based on infection mechanisms, KEDRF is

45 expected to have several advantages, such as potential of responding to low-dose infection,

46 considering host health, sex, pathogen strain, and variability.

47 Few studies have focused on developing dose-response models based on infection

48 mechanisms or key event models describing pathogen response in humans. Koseki et al. ${ }^{3}$

49 and Takeoka et al. (in submission) developed key event models that dynamically describe 
50 the death of some pathogens in simulated gastric fluid mimicking stomach digestion. Pujol

51 et al. ${ }^{4}$ described immune capacities until the occurrence of infection. Pathogens, such as

52 Listeria, Salmonella, and Campylobacter, adhere to and invade intestinal epithelial cells

53 and cause disease ${ }^{5}$. Caco- 2 cells are commonly used to observe the adhesion and invasion

54 of pathogens to intestinal epithelial cells in vitro. We recently developed a model

55 describing the invasion kinetics of pathogens in human intestinal cells ${ }^{6}$. Although one

56 study has previously estimated the dose-response relationship of Listeria through

57 mathematical modeling of bacterial colonization in the human intestine after reductions in

58 the human stomach ${ }^{7}$, this mechanistic dose-response model does not consider cell invasion

59 by the pathogen in terms of infection and that pathogen colonization in the intestines does

60 not always cause infection. The final dose-response relationship needs to describe illness

61 and modeling competitions with the immune system after the invasion of tissues by

62 pathogens and the onset of illness. In these respects, KEDRF is still a developing concept.

63 It is desirable to develop a more sophisticated key events dose-response model to further

64 elucidate the reality of foodborne illness.

65 It is necessary to incorporate the concept of bioaccessibility in KEDRF to develop a

66 more sophisticated mechanistic dose-response model. The term "bioaccessibility," along

67 with "bioavailability," is a key concept to ascertain nutritional efficiency of food and food

68 formulas developed to improve human health in terms of pharmacokinetics and nutrition ${ }^{8}$.

69 Bioaccessibility is defined as the number of chemicals or nutrients that are released from

70 the gastrointestinal tract tissue, which are made available through blood vessels via

71 absorption. It is evaluated using in vitro digestion models, generally simulating gastric or 
72 small intestinal digestion, as revealed through a Caco-2 cell uptake test ${ }^{9}$. Infectious

73 foodborne pathogens invade the tissues through the stomach and intestines, causing

74 inflammation in the gastrointestinal tract, or travel to the affected area, such as blood or

75 lymph, and cause symptoms. Pathogens also trigger physiological reactions through the

76 same pathways as nutrients and chemicals (perhaps slightly oversimplified). At present, it is

77 difficult to experimentally replicate and model the competition between pathogens and

78 immune cells, but the pathways for invading intestinal tissues have been reproduced in

79 vitro. Assessing the bioaccessibility of pathogens is essential to assume a dose-response

80 relationship with $\mathrm{KEDRF}$ as a reference.

81 This study aimed to develop an alternative dose-response model of Campylobacter

82 jejuni, which is one of the most dangerous pathogenic bacteria, based on KEDRF,

83 considering food type and host age. We estimated the invasion probability, defined as the

84 probability of infection, accounting for food type in the gastric retention time and age in the

85 gastric $\mathrm{pH}$. The estimated infection probability was compared and validated using actual

86 epidemiological data.

\section{$87 \quad$ Results}

\section{Gastric bacterial reduction}

89 Changes in the $\mathrm{pH}$ of young and elderly individuals could be successfully described using

90 an exponential model (Fig. 2). All the estimated parameters of the exponential model were

91 convergent for $\mathrm{pH}$ changes after liquid and solid meals among young and elderly

92 individuals (Supplementary Fig. S2) because the Gelman-Rubin convergence statistic (R-

93 hut value) of parameter distributions was 1.0. The estimation of the fitted exponential 
94 model indicated that the changes in the $\mathrm{pH}$ in the stomachs of elderly individuals were

95 broader than those of young individuals (Fig. 2). Differences in the reduction behavior of

96 C. jejuni were shown to be due to differences in $\mathrm{pH}$ (Fig. 3). The calculated survival ratio

97 of elderly individuals after ingestion of liquid and solid foods was higher than that of the

98 calculated survival transit ratio of young individuals.

99

100 Bacterial transfer to the small intestine

101 The changes in the retention ratio in the stomach for solids and liquids could be

102 successfully described as a cumulative gamma distribution (Fig. 4). Estimated parameter

103 distributions converged with Bayesian inference because all R-hut values of parameter

104 distributions were 1.0 (Supplementary Fig. S3). The mean ( \pm standard deviation) time of

105 estimated gastric retention of solid foods was $1.5 \pm 0.52 \mathrm{~h}$, and the mean of estimated

106 gastric retention of liquid foods was $0.77 \pm 0.84 \mathrm{~h}$. The survival ratio of intestinal transit

107 varied with food type and age (Fig. 5). There was a significant difference in the survival

108 transit ratio in young and elderly people after eating solid food. While, there was not

109 notably difference between survival transit ratio of young and elderly individuals after

110 eating liquid foods (Fig. $5 \mathrm{a}$ and b). In addition, considering food type difference, there was

111 a difference in the estimated survival pathogen transit ratio regardless of age (Fig. 5).

113 Retention time in the small intestine

114 The changes in the colonic filling ratio could be successfully described as a cumulative

115 gamma distribution (Fig. 6). The estimated parameter distributions converged with 
116 Bayesian inference since all R-hut values of parameter distributions were 1.0

117 (Supplementary Fig. S4). The mean time of estimated intestinal retention was $5.8 \pm 2.0 \mathrm{~h}$.

119 Probability of infection in human intestinal cells

120 The estimated cell invasion probability of $C$. jejuni varied with food type and age (Fig. 7).

121 There were no differences in the prediction of infection probability among the strains

122 (Supplementary Fig. S7, S8, and S9). The prediction of the infection probability of all three

123 C. jejuni strains is shown in Fig. 7. Young and elderly individuals consuming liquid food

124 (liquid-young and liquid-elderly, respectively), as well as elderly individuals consuming

125 solid food (solid-elderly) estimated infection probabilities were similar to the reported

126 dose-response relationships with bovine milk ${ }^{10}$, and all three strains' $95 \%$ prediction bands

127 covered the reported dose-response relationship. The root mean square error (RMSE) of the

128 median prediction of young-liquid, elderly-solid, and elderly-liquid groups were 0.69, 0.84,

129 and $0.21 \log \mathrm{CFU}$, respectively, when the logarithms of pathogen dose were assumed as

130 objective variables. In contrast, the RMSE of the median prediction of the young-solid

131 group was $1.2 \log \mathrm{CFU}$ and the $95 \%$ prediction band did not cover the reported dose-

132 response relationship.

134 Sensitivity analysis of the framework for estimating invasion probability

135 Figure 8 shows the Spearman's rank correlation coefficients of model components (e.g.,

136 food type, model parameters, $N_{\text {intestine }}$, and $t_{\text {intestinal }}$ ) against the infection probability. 
137 The upper factors in Fig. 8 were more relevant to the estimated infection probability. The 138 indicators of liquid and solid food were set as 0 and 1 , respectively, and the indicators of 139 the strains were set as follows: RIMD 0366027, 1; RIMD 0366042, 2; and RIMD 0366048, 1403 . The indicators for age were set as the mean age of individuals subjected to the $\mathrm{pH}$ test 141 (young: 25; elderly: 71). The most relevant factor against the infection probability was the 142 cell-invading pathogen count $\left(R: 0.96 ; p\right.$-value $\left.<10^{-6}\right)$. The second position of the relevant 143 factor was the logarithm of pathogen count intake $\left(R: 0.90 ; p\right.$-value $\left.<10^{-6}\right)$. Since the 144 infection probability was directly derived from these two factors, it is natural that these 145 factors have the most relevance. The third position of the relevant factor was the intestinal 146 survival ratio $\left(R: 0.29 ; p\right.$-value $\left.<10^{-6}\right)$. The relevant factors from the first to the third place 147 were computable. The most important factors in the parametric factors were the shape 148 parameter of the gamma distribution for gastric retention $\left(R:-0.23 ; p\right.$-value $\left.<10^{-6}\right)$, the 149 second factor was the scale parameter of the gamma distribution for gastric retention $(R:-$ $1500.23 ; p$-value $\left.<10^{-6}\right)$, and the third factor was food type $\left(R:-0.23 ; p\right.$-value $\left.<10^{-6}\right)$. The 151 factors with a $p$-value more than 0.05 were intestinal retention $\alpha$ ( $p$-value: 0.06 ), intestinal 152 retention $\beta$ ( $p$-value: 0.11 ), stomach reduction $b$ ( $p$-value: 0.39 ), intestinal retention time 153 ( $p$-value: 0.70 ), invasion ratio ( $p$-value: 0.70$)$, invasion $\log N_{\max }$ ( $p$-value: 0.96 ), and strain 154 (p-value: 0.99). 


\section{Discussion}

158 Here, this study aimed to develop an alternative dose-response model of $C$. jejuni, one 159 of the most dangerous pathogenic bacteria, through KEDRF, considering food type and 160 host age. Despite the completely different approach to estimating the dose-response

161 relationship using conventional methods, the predictive model developed in this study

162 successfully predicted the reported illness probability of campylobacteriosis ${ }^{10}$. The 163 reported dose-response relationship (illness probability) resulted from contaminated milk 164 consumption, which could be properly predicted from among predictions of the KEDRF 165 dose-response model for both young and elderly individuals ingesting liquid foods.

166 However, it should be noted that there were small but unignorable RMSEs of young and 167 elderly individuals ingesting liquid foods ( 0.69 and $0.84 \log \mathrm{CFU}$, respectively) with a 168 reported dose-response relationship. The infection probability according to the dose169 response relationship estimated in this study was based on the probability of invasion into 170 intestinal cells. Because the immune system prevents symptoms of the illness after the 171 invasion of the intestinal tissue, the illness probability being lower than the infection 172 probability is natural.

173 The predicted results of this study showed that $C$. jejuni could invade the intestinal 174 tissues and infect the human body even at a dose as low as 1-10 CFU. The previously 175 reported epidemiological data on milk consumption also exhibited the occurrence of the 176 disease at low doses. In contrast, the dose-response of $C$. jejuni demonstrated herein 177 showed a discrepancy with the previously reported model ${ }^{11}$, which is also the most widely 178 used dose-response relationship for C. jejuni in QMRA. The infection probability reported 
179 by Black et al. ${ }^{11}$ was based on the presence of $C$. jejuni in stools, the definition of which 180 was completely different from that examined in this study. The difference in definition 181 might be the reason for the difference in the prediction results. It has been reported that $C$. 182 jejuni growth is inhibited upon competition with the extended-spectrum $\beta$-lactamase183 producing bacteria, including some strains of Escherichia coli, which are widespread in 184 nature ${ }^{12}$. In the colon, which is not an optimum environment for $C$. jejuni because of the 185 anaerobic environment, the number of viable $C$. jejuni may decrease owing to competition 186 with other intestinal bacteria. $C$. jejuni may be reduced before it is detected in the stool 187 owing to competition for nutrients or competitive effects in the gut, such as the Jameson 188 effect ${ }^{13}$. Considering the actual behavior of $C$. jejuni in the human body, it is better to 189 discuss the illness probability or the invasion probability into human tissues, which is the 190 definition used for the probability of infection in this study.

191 While the results for the prediction were similar to those reported for actual dose192 response relationships ${ }^{10}$, it was also possible to show the effectiveness of the 193 implementation of the computational framework based on KEDRF. In particular, the results 194 will be considered very beneficial, because the dose-response relationship depends on the 195 food type and the age of the host. The FAO/WHO risk assessment for Listeria also 196 emphasizes the importance of dose-response relationships in elderly and high-risk 197 populations ${ }^{14}$. In particular, the results of this study indicated a large difference in the 198 infection probability (Young: $1.9 \log$ CFU; Elderly: $1.1 \log$ CFU) among the food types. In 199 the sensitivity analysis, the factor related to the retention time in the stomach had the largest 200 correlation among the parameters. Figure 5 shows that when consuming liquid food, the 
201 number of $C$. jejuni reaching the intestines was higher than that when consuming solid food

202 owing to the difference in the gastric retention time. The difference in the predicted

203 probability of infection between solid and liquid foods was due to the difference in the

204 gastric retention time. In contrast, the predictions did not show any difference among

205 pathogen strains. In the present dose-response model, the strains were reflected only in the

206 invasion into the intestinal tissue. Although there was no strain difference in the invasion

207 behavior, there could be differences in some other key events, such as survival in the

208 stomach. KEDRF has the potential to estimate the probability of infection in young

209 children since the difference between the elderly and young individuals was considered in

210 this study. KEDRF, which can simultaneously consider various conditions, such as host and

211 food type, would be a useful tool for estimating dose-response relationships.

212 Key event models using Bayesian inference are important in KEDRF, where

213 predictions are chained for each key event. This study attempted to illustrate the variability

214 and uncertainty of pathogen behavior and the environment of the gastrointestinal tract

215 based on Bayesian inference. Modeling using Bayesian inference has been used to describe

216 various bacterial behaviors, such as the growth and death of various bacteria, as a method

217 that can represent variability and uncertainty ${ }^{6,15-17}$. In addition, KEDRF suggests that

218 modeling the individual variability of the digestive process in different hosts will lead to a

219 better understanding of food poisoning incidents. The use of Bayesian inference to

220 represent not only bacterial but also host variability will allow the estimation of appropriate

221 dose-response relationships using mechanistic approaches. 
Although this study has shown that KEDRF is a useful procedure for predicting

223 dose-response relationships of $C$. jejuni, KEDRF is also effective for other types of

224 bacteria. The approach used in this study consisted of a mathematical prediction model

225 based on predictive microbiology and pharmacokinetics. The growth and death of C. jejuni,

226 as well as various other pathogens, were described using predictive models. For many other

227 pathogens, the present method can be applied to calculate the intestinal viable bacterial

228 count using gastric retention time and survival kinetics in the stomach, independent of the

229 pathogen type. Development of dose-response models based on KEDRF is expected for

230 various foodborne pathogens.

231 However, the dose-response model based on KEDRF presented in this study still

232 has certain limitations. The present study did not consider the growth of $C$. jejuni in the

233 intestines because its growth rate is slower than its invasion rate ${ }^{6}$. In contrast, a growth

234 model is needed for fast-growing pathogenic bacteria, such as E. coli or Salmonella.

235 Modeling the interactions between the immune system and pathogens is also required. It

236 has been suggested that the probability of infection may be high, but the illness may not

237 develop ${ }^{18}$. Modeling the effect of immunity on pathogens will be necessary to predict the

238 probability of illness. Furthermore, since the available data on the gastrointestinal tract

239 were limited, the effect of age was reflected only in the $\mathrm{pH}$ change and the effect of food

240 type was reflected only in the residence time in the stomach in this study. However, for a

241 more realistic prediction, data corresponding to age and food types in all gastrointestinal

242 environments, such as $\mathrm{pH}$ change, gastric retention time, and intestinal retention time, are

243 needed. For more realistic and appropriate predictions of the dose-response relationship 
244 based on KEDRF, it is necessary to study immune modeling and additional environmental 245 data of the gastrointestinal tract under various conditions.

246 In conclusion, the behavior of $C$. jejuni in the gastrointestinal tract based on the

247 KEDRF was successfully predicted via mathematical models using Bayesian inference.

248 Moreover, the respective dose-response relationships for combinations of age (young,

249 elderly) and food type (liquid, solid) were also estimated. The results of the dose-response

250 model of KEDRF showed similar results to the reported dose-response relationship.

251 Furthermore, sensitivity analysis of the prediction results showed that gastric retention time

252 was the most relevant factor among the key events from ingestion to invasion. This study

253 demonstrated a large potential for the development of a novel dose-response model based

254 on KEDRF. The dose-response model based on KEDRF will allow us to estimate the dose-

255 response relationships of various pathogens with various factors, such as age, sex, chronic

256 illness, food type, and others based on their actual infection mechanisms.

258 Methods

259 Determining the key events of campylobacteriosis infection

260 The key events of the infection mechanism were ascertained on the basis of the KEDRF

261 report ${ }^{1}$. Since it is difficult to quantitatively assess pathogens in the human body, this study

262 considered the probability of invasion into the small intestinal endothelial cells as the

263 infection probability. The following were identified as key events: (i) pathogen reduction in

264 the stomach; (ii) transfer to the small intestine from the stomach; (iii) pathogen invasion

265 into small intestinal epithelial cells. The growth of $C$. jejuni in the small intestine was not 
266 considered because a preliminary test indicated that it was relatively slow compared to the

267 invasion rate of the epithelial cells ${ }^{6}$. Figure 1 shows the constructed model, and the

268 abbreviations are summarized in Supplementary Table S1. All the computations were

269 calculated under the Anaconda distribution (Python 3.7.7) (See data availability section).

271 Modeling for postprandial gastric pH change among younger and elder individuals

272 The postprandial pH changes among young and elderly individuals were expressed

273 separately in a mathematical model. The exponential models (Eq. 1) were fitted to the

274 reported $\mathrm{pH}$ changes after a standard meal $(1000 \mathrm{kcal})$ for young ${ }^{19}$ and elderly individuals $275 \quad 20$ :

$$
p H_{(t)}=p H_{0} e^{-k_{p H} t}+p H_{\min }
$$

277 where $p H_{(t)}$ denotes the $\mathrm{pH}$ at a time after food intake, $t ; p H_{0}$ denotes $\mathrm{pH}$ immediately

278 after a meal; $k_{p H}$ denotes the decreasing rate of $\mathrm{pH} ; p H_{\min }$ denotes the convergence value

279 of $\mathrm{pH}$. The parameters were estimated using Bayesian inference through pystan (ver. 2.19.).

280 The normal distribution, which is generally used, was adopted as the prior distribution of

$281 \mathrm{pH}$, as distributions of the reported data were contrasting.

282

283 Pathogen survival in stomach with between- and within-strain variability

284 The survival of $C$. jejuni was described using a previously reported dynamic survival model 285 of C. jejuni (Eq. 2) under artificial gastric conditions using Bayesian inference (Takeoka et 286 al., in submission). The between- and within-strain variability reduction model was

287 constructed from the data of 11 strains of $C$. jejuni (RIMD 0366026, RIMD 0366027 , 
RIMD 0366028, RIMD 0366029, RIMD 0366042, RIMD 0366043, RIMD 0366044 ,

RIMD 0366048, RIMD 0366049, RIMD 0366050, and RIMD 0366051).

$$
\log _{10} S_{g(t+\Delta t)}=-\left(\frac{t^{*}+\Delta t}{\delta_{(\overline{p H})}}\right)^{p_{(\overline{p H})}}
$$

$$
t^{*}=\delta_{(\overline{p H})}\left(-\log _{10} S_{g(t)}\right)^{\frac{1}{p}}
$$

$$
\left\{\begin{array}{l}
\ln \left(\delta_{(\overline{p H})}\right)=a \times p H+b \\
\ln \left(p_{(\overline{p H})}\right)=e \times p H+f
\end{array}\right.
$$

$$
\left(\begin{array}{l}
a \\
b \\
e \\
f
\end{array}\right) \sim \text { MultiNormal_Cholesky }\left(\left(\begin{array}{l}
a_{0} \\
b_{0} \\
e_{0} \\
f_{0}
\end{array}\right), \Sigma_{\text {chol }}\right)
$$

295 where $S_{g(t)}$ denotes the bacterial survival ratio defined as the ratio of the surviving bacterial 296 counts divided by the initial bacterial counts; $p$ denotes the power parameter of the Weibull 297 model; $\delta$ denotes the time of the first decimal reduction of the Weibull model; and $\overline{p H}$

298 denotes the mean $\mathrm{pH}$ during the time intervals from $t$ to $t+\Delta t$ as $\overline{p H}=\frac{p H_{(t)}+p H_{(t+\Delta t)}}{2}$. The 299 parameters of the primary model, $\delta$ and $p$, were defined using the parameters of the secondary 300 model $(a, b, c, f)$, following the multivariate normal distribution of Cholesky parameterization, in 301 which $\Sigma_{\text {chol }}$ is the Cholesky factor of the covariance matrix of $\log \left(p_{(\overline{p H})}\right)$ and $\log \left(\delta_{(\overline{p H})}\right)$ of all 302 strains. 


\section{Bacterial transfer to the small intestine}

305 Changes in the gastric retention ratio were described as the cumulative gamma distribution, 306 which is used to describe the waiting time of traffic jams and transmitting times.

307 Comparing the transfer time of solid foods and liquid foods, the effect on the cell invasion 308 ability due to differences in food type was estimated. The changes in gastric retention ratio 309 were described using the following cumulative gamma distribution fitted to the reported 310 change in the gastric retention ratio after solid and liquid meals: ${ }^{21}$

$$
R_{g}=1-\frac{1}{\Gamma_{(\alpha)}} \gamma_{(\alpha, \beta t)}
$$

312 where $R_{g}$ is the gastric retention ratio; $\alpha$ is the shape parameter; $\beta$ is the rate parameter of

313 gamma distribution; $\Gamma_{(\alpha)}$ is the gamma function $\Gamma_{(\alpha)}=\int_{0}^{\infty} e^{-u} u^{\alpha-1} d u ; \gamma_{(\alpha, \beta t)}$ is the lower

314 incomplete gamma function $\gamma_{(\alpha, \beta t)}=\sum_{k=0}^{\infty} \frac{(\beta t)^{\alpha+k} e^{-\beta t}}{\alpha(\alpha+1) \ldots(\alpha+k)}$.

315 The combined equation of $\mathrm{pH}$, pathogenic survival, and gastric retention model, as well

316 as the ratio of the surviving pathogen transfer to the intestine, $r_{\text {intestine }}$, was described (Eq.

317 4; graphically description: Supplementary Fig. S1), and discretely expressed as shown in

318 Eq. 4.b:

319

$$
\begin{aligned}
& r_{\text {intestine, }(t)}=\int_{0}^{t} S_{g(s)} \frac{d R_{g}}{d s} d s \\
& r_{\text {intestine, }\left(t_{k}\right)}=\sum_{i=1}^{k} \frac{S_{\left(t_{i}\right)}}{n}
\end{aligned}
$$

321 where $s$ denotes an operator; $t_{i}$ denotes the simulated gastric retention times from the 322 gamma distributions; $n$ denotes the total simulation counts from the gamma distributions; $k$ 
323 denotes any natural number between 1 and $n$. Using Eq. 4, the survival pathogen transit

324 counts, $N_{\text {intestinal }}$, were derived as follows:

$325 \quad N_{\text {intestine }}=r_{\text {intestine }} N_{\text {dose }}$

326 where $N_{\text {dose }}$ denotes the intake counts of pathogens.

327

328 Bacterial invasion in intestinal cells

329 Invasion of C. jejuni was described using a modified predictive model based on a 330 previously reported $C$. jejuni model $^{6}$ (RIMD 0366027, RIMD 0366042, and RIMD

331 0366048) applying invasion count, $N_{\text {invading }}$, to Caco-2 cells as follows:

332

$\frac{d}{d t} N_{\text {invading }}=\mu \frac{N_{\text {exposure }}-N_{\text {invading }}}{V}\left(S N_{\max }-N_{\text {invading }}\right)$

333 where $\mu, N_{\text {exposure }}, N_{\max }, V$, and $S$ denote the cell invasion rate, the pathogen exposure

334 count, the spatial maximum invading pathogen count per $1 \mathrm{~cm}^{2}$, the volume of intestinal 335 juice $(319 \mathrm{~mL})^{22}$ and the surface area of the small intestine $\left(32 \mathrm{~m}^{2}\right)$, respectively ${ }^{23}$.

337 Retention time in the small intestine

338 The cumulative gamma distribution was fitted to the change in the small intestinal retention

339 ratio, $R_{\text {intestinal }}$, as the reported colonic filling ratio was as follows ${ }^{24}$ :

340

$$
R_{\text {intestinal }}=1-\frac{1}{\Gamma_{(\alpha)}} \gamma_{\left(\alpha^{\prime}, \beta^{\prime} t\right)}
$$

341 Considering Eq. 7, small intestinal retention time, $t_{\text {intestinal }}$, follows the gamma

342 distribution $\alpha^{\prime}$ as a shape parameter and $\beta^{\prime}$ as a rate parameter; $\operatorname{Gamma}\left(\alpha^{\prime}, \beta^{\prime}\right)$ as follows:

343

$$
t_{\text {intestinal }} \sim \operatorname{Gamma}\left(\alpha^{\prime}, \beta^{\prime}\right)
$$




\section{Invasion probability in human tissues}

346 The probability of pathogen invasion into the cells was determined as previously described

$347{ }^{6}$. One or more $C$. jejuni cell invasion probabilities were derived from the models as

348 follows:

349

$$
P_{\text {invading }}=1-\left(1-\frac{N_{\text {invading }\left(t_{\text {intestinal }}\right)}}{N_{\text {Dose }}}\right)^{N_{\text {Dose }}}
$$

350 Spearman's rank correlation coefficients between $P_{\text {invading }}$ and the parameters were

351 established as a sensitivity analysis of the current dose-response model.

352 


\section{References}

354 1. Buchanan, R. L., Havelaar, A. H., Smith, M. A., Whiting, R. C. \& Julien, E. The key events

355 dose-response framework: its potential for application to foodborne pathogenic

356 microorganisms. Crit. Rev. Food Sci. Nutr. 49(8), 718-728 (2009).

357 2. Julien, E., Boobis, A. R., Olin, S. S. \& Ilsi Research Foundation Threshold Working Group.

358 The key events dose-response framework: a cross-disciplinary mode-of-action based

359 approach to examining dose-response and thresholds. Crit. Rev. Food Sci. Nutr. 49(8), 682-

$360 \quad 689(2009)$.

361 3. Koseki, S., Mizuno, Y. \& Sotome, I. Modeling of pathogen survival during simulated gastric

362 digestion. Appl. Environ. Microbiol. 77(3), 1021-1032 (2011).

363 4. Pujol, J. M., Eisenberg, J. E., Haas, C. N. \& Koopman, J. S. The effect of ongoing exposure

364 dynamics in dose response relationships. PLOS Comput. Biol. 5(6), e1000399;

$365 \quad$ 10.1371/journal.pcbi.1000399 (2009).

366 5. Ribet, D. \& Cossart, P. How bacterial pathogens colonize their hosts and invade deeper 367 tissues. Microbes Infect. 17(3), 173-183 (2015).

368 6. Abe, H., Koyama, K. \& Koseki, S. Modeling invasion of Campylobacter jejuni into human 369 small intestinal epithelial-like cells by Bayesian inference. Appl. Environ. Microbiol. 87(1), $370 \quad$ e01551-20; 10.1128/AEM.01551-20 (2020).

371 7. Rahman, A., Munther, D., Fazil, A., Smith, B. \& Wu, J. Advancing risk assessment:

372 mechanistic dose-response modelling of Listeria monocytogenes infection in human 373 populations. R. Soc. Open Sci. 5(8), 180343; 10.1098/rsos.180343 (2018).

374 8. Fernández-García, E., Carvajal-Lérida, I. \& Pérez-Gálvez, A. In vitro bioaccessibility 375 assessment as a prediction tool of nutritional efficiency. Nutr. Res. 29(11), 751-760 (2009).

376 9. Galanakis, C. M. Nutraceutical and Functional Food Components (Academic Press, 2016) 
377 10. Teunis, P. et al. A reconsideration of the Campylobacter dose-response relation. Epidemiol. $378 \quad$ Infect. 133(4), 583-592 (2005).

379 11. Black, R. E., Levine, M. M., Clements, M. L., Hughes, T. P. \& Blaser, M. J. Experimental 380 Campylobacter jejuni infection in humans. J. Infect. Dis. 157(3), 472-479 (1988).

381 12. Hazeleger, W. C., Jacobs-Reitsma, W. F. \& den Besten, H. M. W. Quantification of Growth of Campylobacter and extended spectrum $\beta$-lactamase producing bacteria sheds light on black box of enrichment procedures. Front. Microbiol. 7, 1430; 10.3389/fmicb.2016.01430

385 13. Jameson, J. E. A discussion of the dynamics of salmonella enrichment. J. Hyg. (Lond). 60, $386 \quad$ 193-207 (1962).

387 14. Food \& Agriculture Organization. Risk Assessment of Listeria monocytogenes in Ready-to$388 \quad$ Eat Foods, 2004

389 15. Koyama, K., Aspridou, Z., Koseki, S. \& Koutsoumanis, K. Describing uncertainty in 390 salmonella thermal inactivation using Bayesian statistical modeling. Front. Microbiol. 10, $391 \quad 2239 ; 10.3389 /$ fmicb.2019.02239 (2019).

392 16. Huang, L. \& Li, C. Growth of Clostridium perfringens in cooked chicken during cooling: 393 one-step dynamic inverse analysis, sensitivity analysis, and Markov chain Monte Carlo 394 simulation. Food Microbiol. 85, 103285; 10.1016/j.fm.2019.103285 (2020).

395 17. Garre, A., Zwietering, M. H. \& den Besten, H. M. W. Multilevel modelling as a tool to 396 include variability and uncertainty in quantitative microbiology and risk assessment. 397 Thermal inactivation of Listeria monocytogenes as proof of concept. Food Res. Int. 137, 398 109374; 10.1016/j.foodres.2020.109374 (2020).

399 18. Teunis, P. F. M., Bonačić Marinović, A., Tribble, D. R., Porter, C. K. \& Swart, A. Acute 400 illness from Campylobacter jejuni may require high doses while infection occurs at low 
doses. Epidemics 24, 1-20 (2018).

402 19. Dressman, J. B. et al. Upper gastrointestinal (GI) pH in young, healthy men and women. Pharm. Res. 7(7), 756-761 (1990).

404 20. Russell, T. L. et al. Upper gastrointestinal pH in seventy-nine healthy, elderly, North American men and women. Pharm. Res. 10(2), 187-196 (1993).

406 21. Hellström, P. M., Grybäck, P. \& Jacobsson, H. The physiology of gastric emptying. Best Pract. Res. Clin. Anaesthesiol. 20(3), 397-407 (2006).

408 22. Schiller, C. et al. Intestinal fluid volumes and transit of dosage forms as assessed by 409 magnetic resonance imaging. Aliment. Pharmacol. Ther. 22(10), 971-979 (2005).

410 23. Helander, H. F. \& Fändriks, L. Surface area of the digestive tract - revisited. Scand. J. Gastroenterol. 49(6), 681-689 (2014).

412 24. Read, N. W., Al-Janabi, M. N., Holgate, A. M., Barber, D. C. \& Edwards, C. A. 413 Simultaneous measurement of gastric emptying, small bowel residence and colonic filling of 414 a solid meal by the use of the gamma camera. Gut 27(3), 300-308 (1986). 


\section{Acknowledgments}

417 This study was supported by a grant from the Food Safety Commission, Cabinet Office,

418 Government of Japan (Research Program for Risk Assessment Study on Food Safety, No. 1802)

419 and a Grant-in-Aid for Japan Society for the Promotion of Science Fellows, Grant number

420 20J11550).

421

422 Author contributions

423

424 Competing interests

425 The author(s) declare no competing interests.

426

427 Data availability

428 The Python codes are available through co-author GitHub (URL: the link will be paste here after the 429 review).

430 For reviewers link: https://www.dropbox.com/sh/f6tuqo8gx8egglv/AAD-

4319 mic5MYtF76kzNYw11P-a?dl=0

432 


\section{Figure legends}

434 Figure 1. Directed acyclic graph of the model parameters and factors. Solid arrows indicate

435 distributions, dashed arrows deterministic functions. The abbreviations and details of

436 components are summarized in Supplementary Table S1.

438 Figure 2. Reported after-meal pH changes (points) of young (upper) and elderly (lower)

439 people, and the prediction band derived from the exponential model. (Appendix: the

440 MCMC trace plots of parameters and the estimated parameter distribution of $\mathrm{pH}$ model).

441

442 Figure 3. Predicted after-meal survival curves (solid curve: median, dash curve and covered

443 range: $90 \%$ prediction band) of $C$. jejuni in the stomach of young (upper) and elderly

444 (lower) people.

445

446 Figure 4. Reported retention ratio (a; points) \& predicted cumulative gamma distributions

447 (a and b; solid curve: median, dash curve and covered range: 95\% prediction band) for

448 gastric retention time (Appendix: Estimated parameter distribution of retention models).

450 Figure 5. Calculated transferred survival ratio (solid curve: median, dash curve and covered

451 range: $90 \%$ prediction band) in intestine under each condition (a: young and liquid food, b:

452 elderly and liquid food, c: young and liquid food, d: elderly and solid food). 
454 Figure 6. Reported colonic filling ratio (a; points) and predicted cumulative gamma

455 distribution for retention time in small intestines ( $\mathbf{a}$ and $\mathbf{b}$; solid curve: median, dash curve

456 and covered range: 95\% prediction band). (Appendix Estimated parameter distribution of

457 colonic filling)

458

459 Figure 7. Cell invasion probability (solid curve: median, dash curve and covered range:

$46060 \%$ and $95 \%$ prediction band) of $C$. jejuni (total of all three strains) under each condition

461 (Same position as Fig. 5) and the reported dose-response relationship (square; Teunis et al.,

462 2005)

463

464 Figure 8. Spearman's ranked correlation coefficients of parameter and computable factors

465 against the predicted infection probability. 


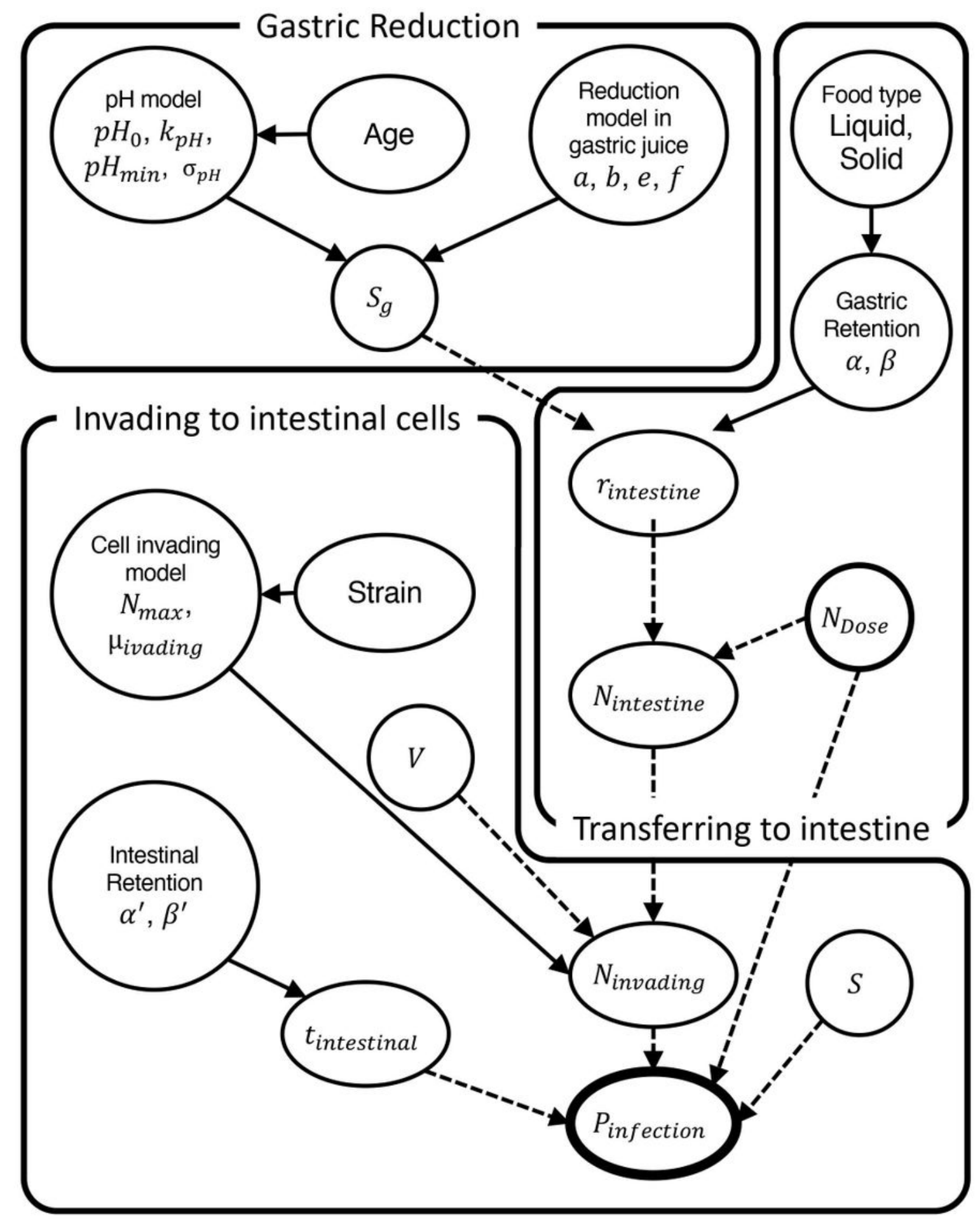

Figure 1

Directed acyclic graph of the model parameters and factors. Solid arrows indicate distributions, dashed arrows deterministic functions. The abbreviations and details of components are summarized in Supplementary Table S1. 

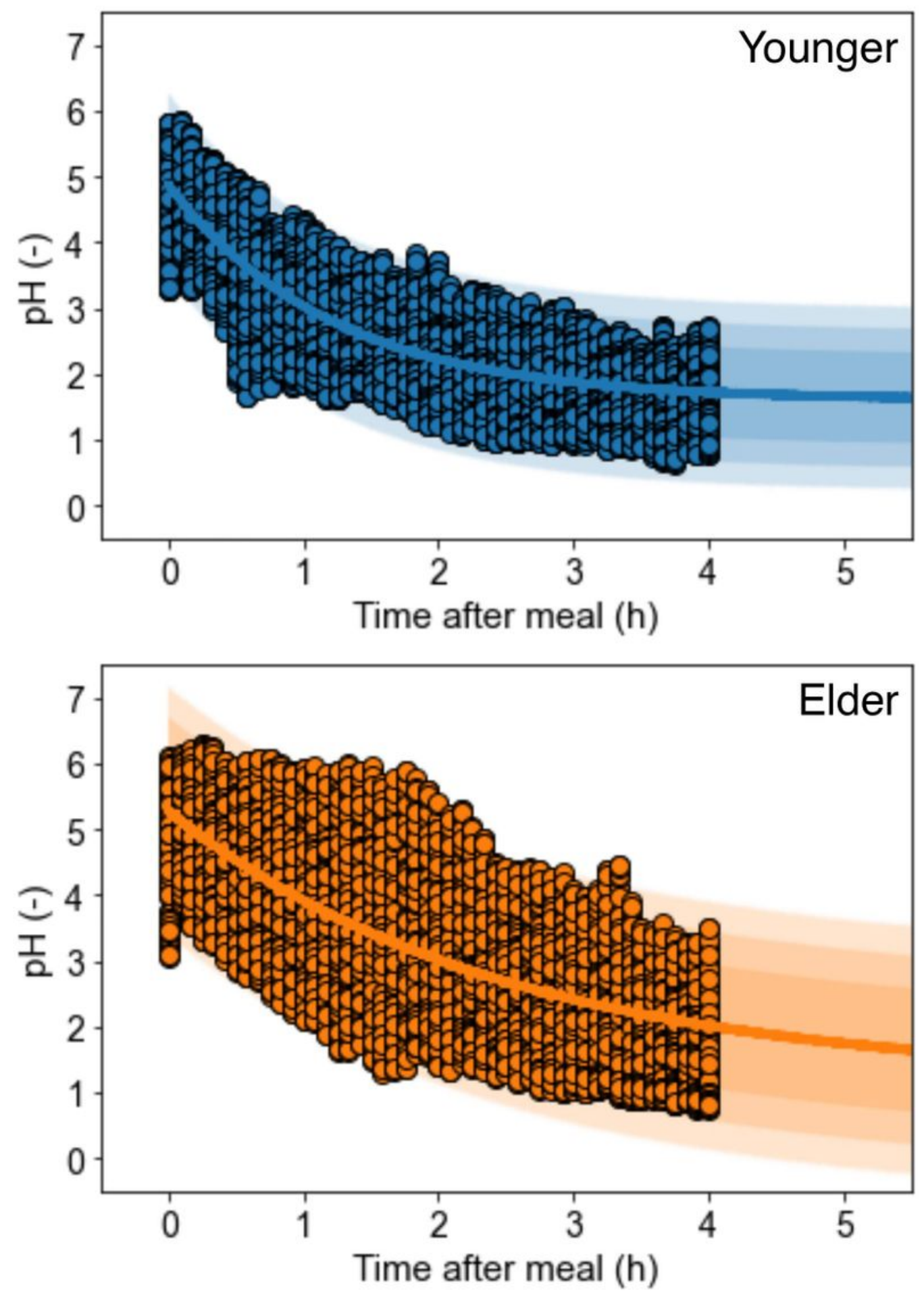

Figure 2

Reported after-meal pH changes (points) of young (upper) and elderly (lower) people, and the prediction band derived from the exponential model. (Appendix: the MCMC trace plots of parameters and the estimated parameter distribution of $\mathrm{pH}$ model). 

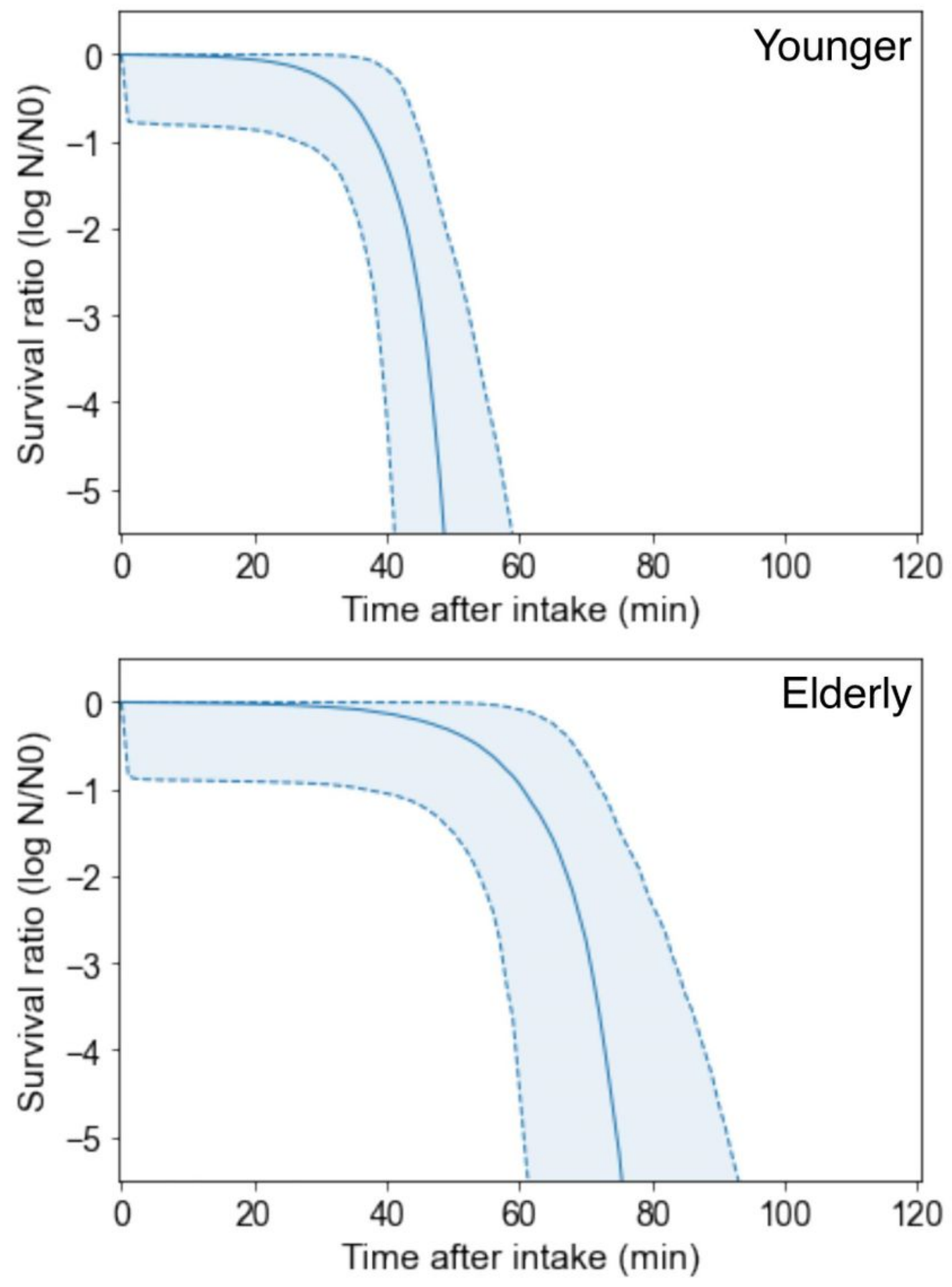

Figure 3

Predicted after-meal survival curves (solid curve: median, dash curve and covered range: $90 \%$ prediction band) of $C$. jejuni in the stomach of young (upper) and elderly (lower) people. 

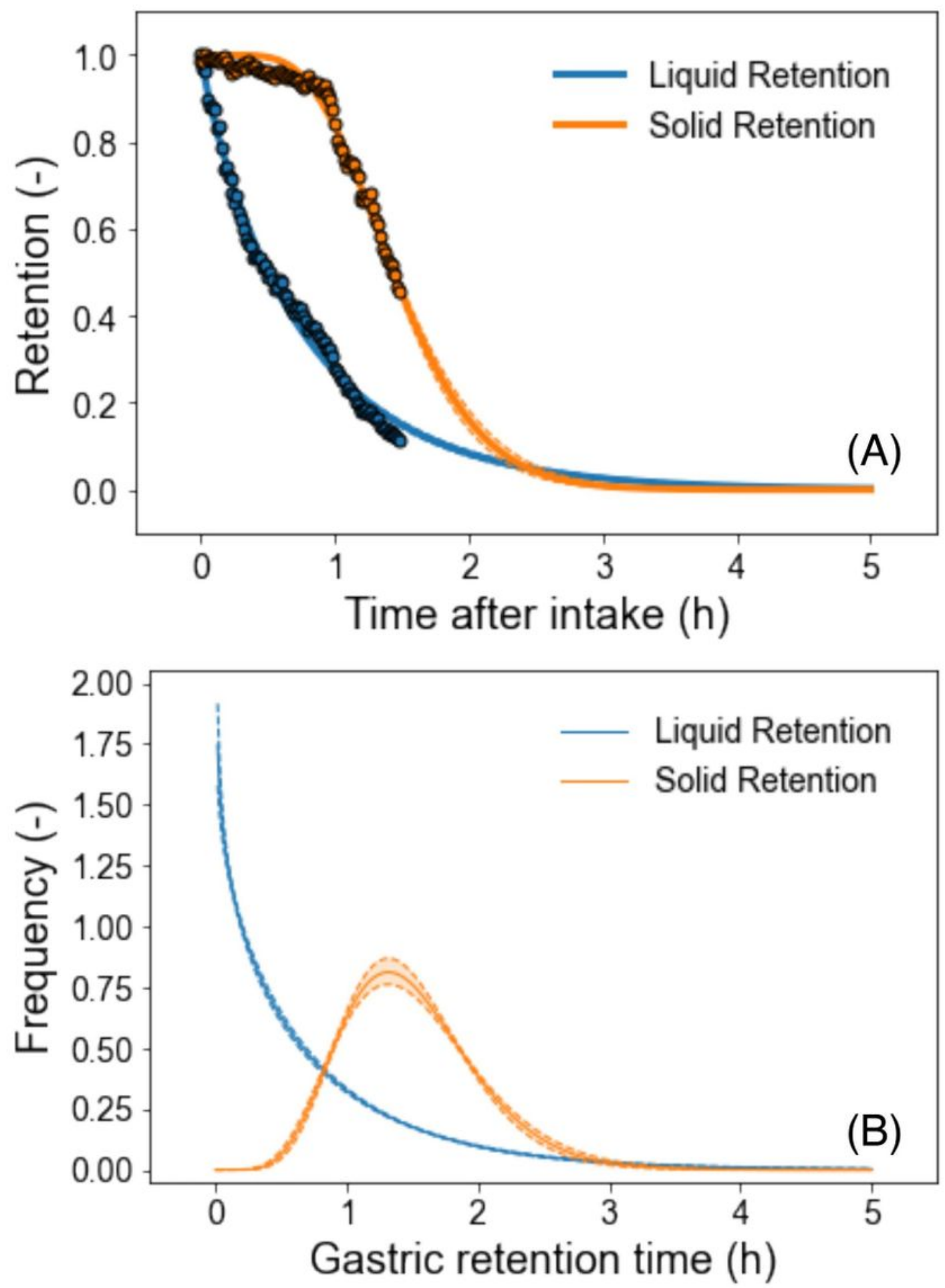

Figure 4

Reported retention ratio (a; points) \& predicted cumulative gamma distributions (a and b; solid curve: median, dash curve and covered range: $95 \%$ prediction band) for gastric retention time (Appendix: Estimated parameter distribution of retention models). 


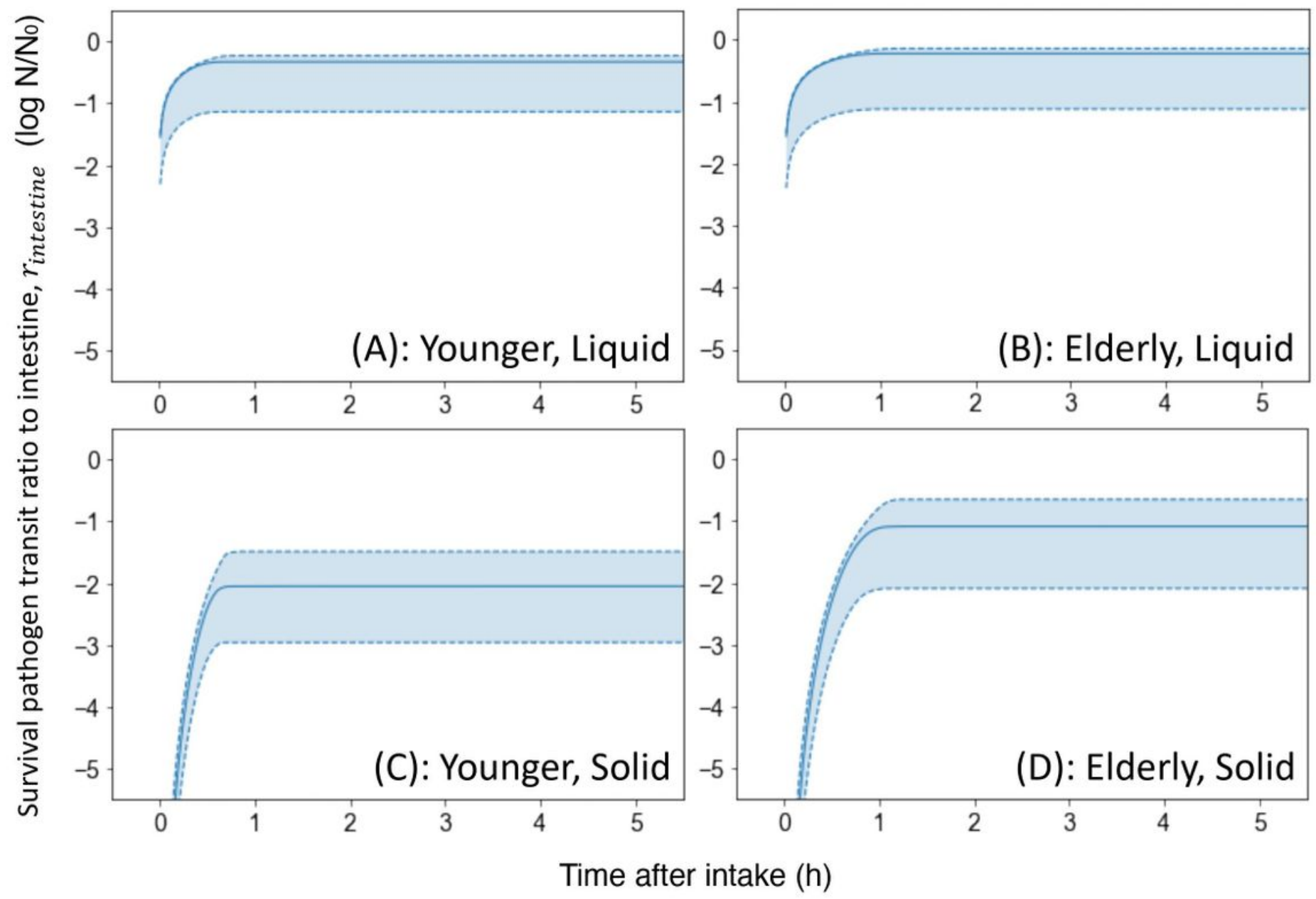

Figure 5

Calculated transferred survival ratio (solid curve: median, dash curve and covered range: $90 \%$ prediction band) in intestine under each condition (a: young and liquid food, b: elderly and liquid food, c: young and liquid food, d: elderly and solid food). 

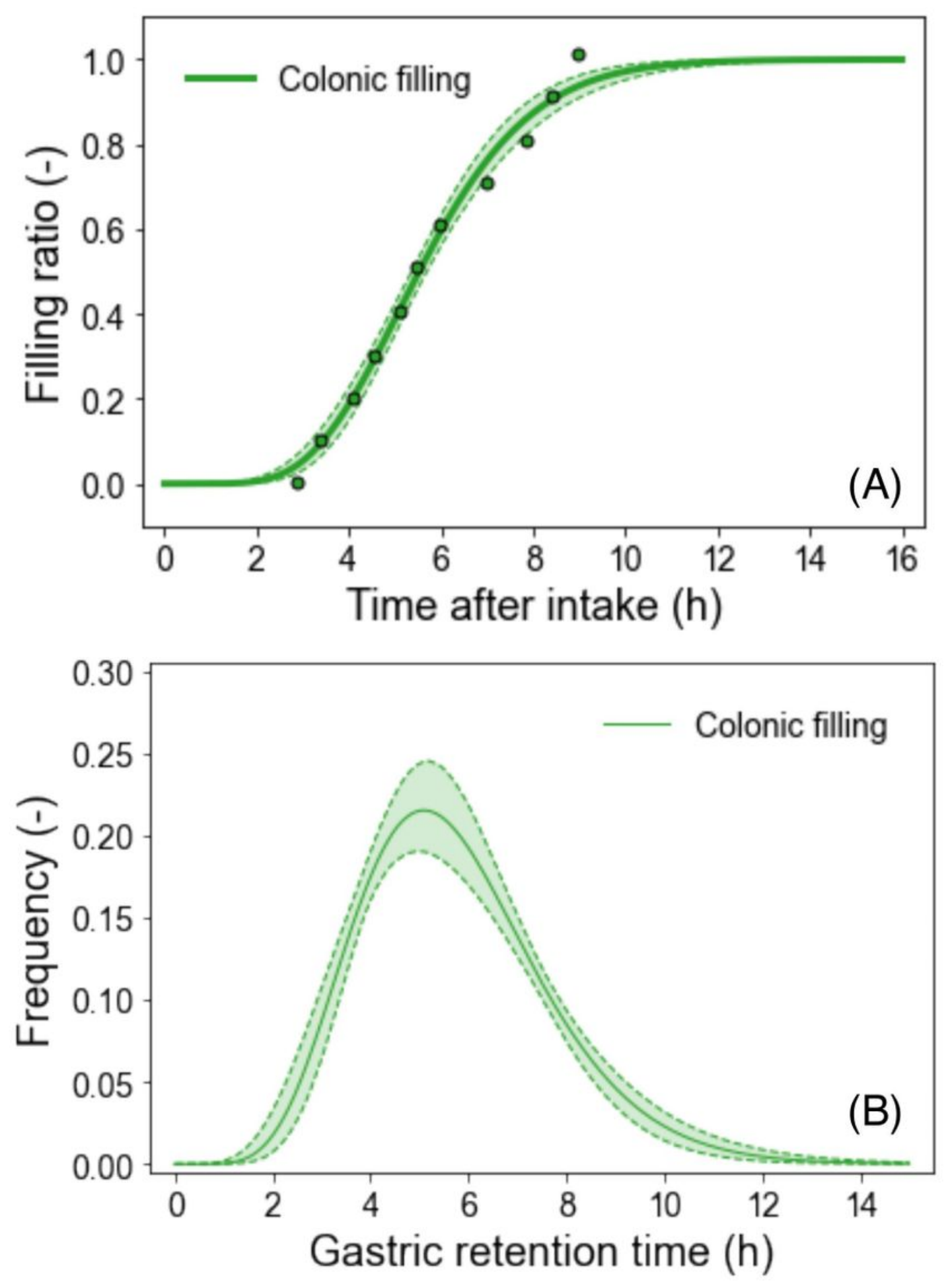

Figure 6

Reported colonic filling ratio (a; points) and predicted cumulative gamma distribution for retention time in small intestines ( $a$ and b; solid curve: median, dash curve and covered range: $95 \%$ prediction band). (Appendix Estimated parameter distribution of colonic filling) 

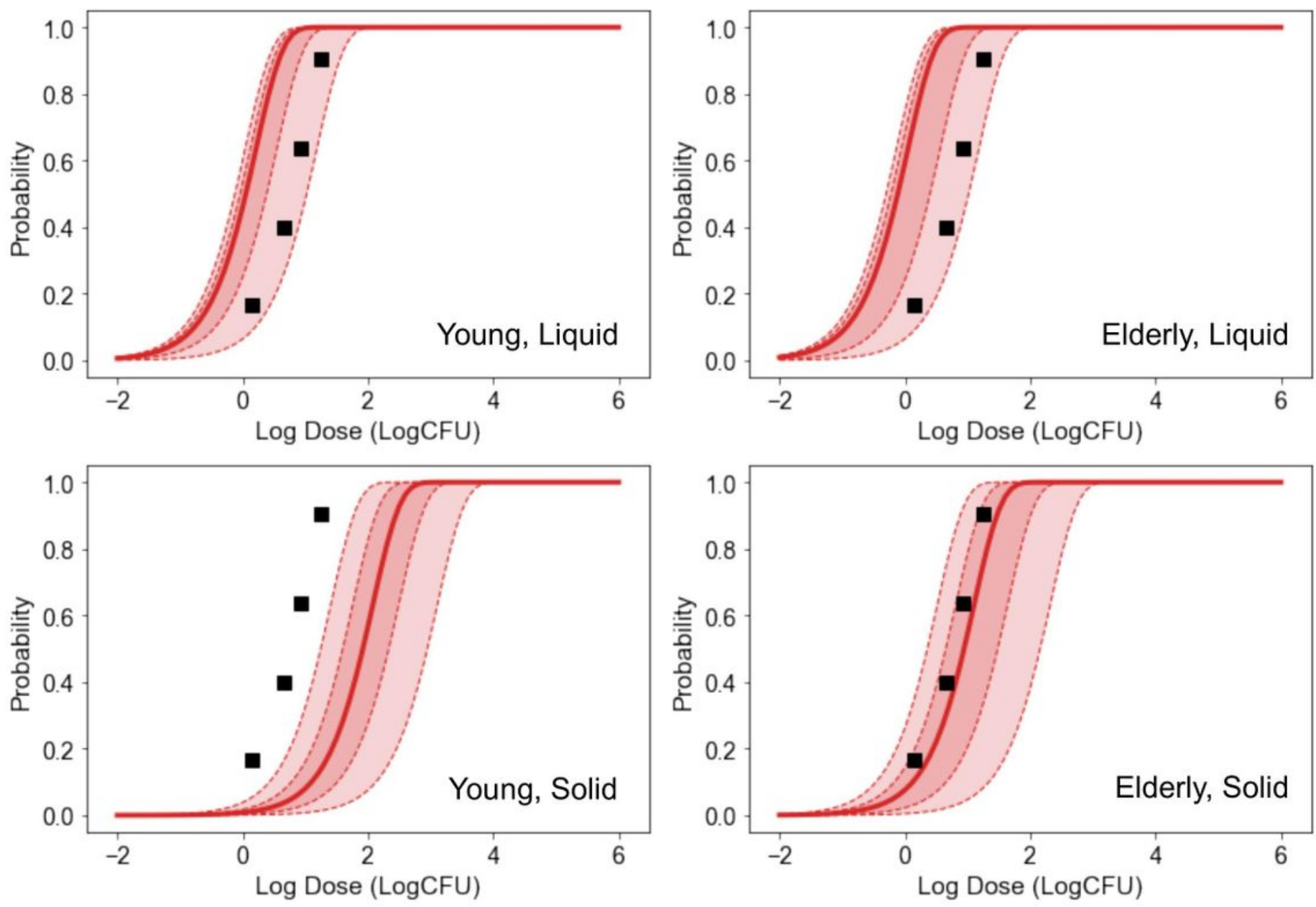

Figure 7

Cell invasion probability (solid curve: median, dash curve and covered range: $60 \%$ and $95 \%$ prediction band) of C. jejuni (total of all three strains) under each condition (Same position as Fig. 5) and the reported dose-response relationship (square; Teunis et al., 2005) 


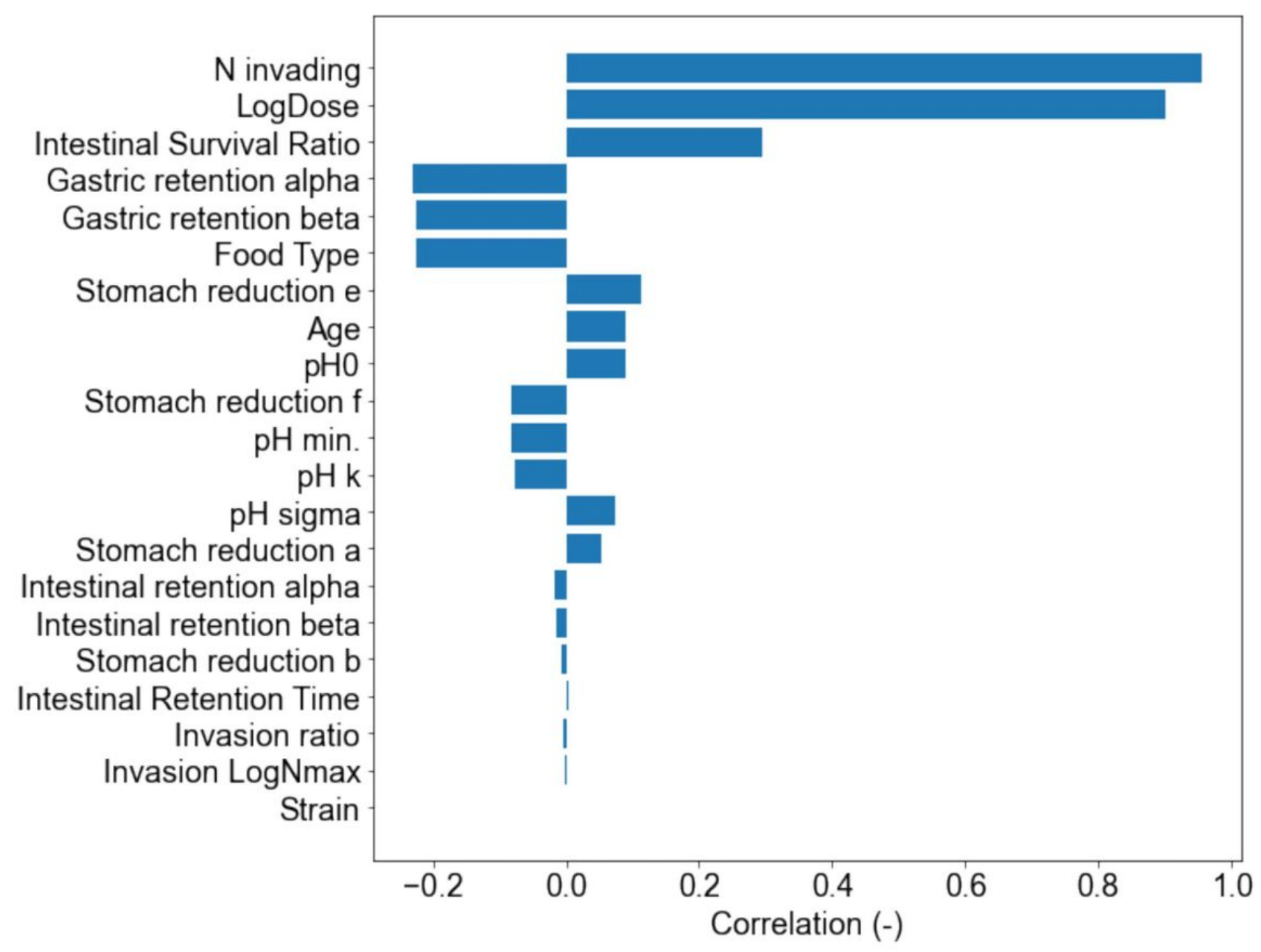

Figure 8

Spearman's ranked correlation coefficients of parameter and computable factors against the predicted infection probability.

\section{Supplementary Files}

This is a list of supplementary files associated with this preprint. Click to download.

- Supplementaryinformation.docx 